\title{
Pharmacoeconomics Applied to Chronic Hepatitis C
}

\author{
Fernando Franciosi Tatsch', \\ Hoel Sette Jr. ${ }^{2}$ and Denizar Vianna ${ }^{3}$
}

\author{
${ }^{1}$ Roche Brazil, ${ }^{2}$ University of São Paulo; São Paulo, SP; ${ }^{3}$ State University \\ of Rio de Janeiro (UERJ); Rio de Janeiro, RJ, Brazil
}

\begin{abstract}
Life expectancy has increased over the last century as it had never been before. This is the result of a combination of many favorable variables such as level of education, improved socio-economic environment and development of medicine. However, new improvements demand heavy investment. Thus, the incorporation of medical technology became a health and economic issue. The pharmacoeconomic knowledge field is being developed to help in the analysis of medical costs and patient needs. The applies to hepatitic $\mathrm{C}$, a common and chronic worldwide disease. In this article, the authors describe the rational behind this type of health economic analysis and review a hepatitis $\mathrm{C}$ model. Overall, in a non-Brazilian scenario, it was demonstrated that peginterferon alfa-2a (40KD) is cost effective in the treatment of $\mathrm{HCV}$ disease.

Key Words: Pharmacoeconomic, hepatitis C, peginterferon alfa-2a.
\end{abstract}

Politically organized societies present demands that correspond to a collective set of individual needs. Among these demands, health occupies a very important position. Its maintenance has continuously required increases in resources. However, most of the individuals involved remain unsatisfied.

Health professionals are underpaid, health insurance companies complain about resource overuse, hospitals and clinics often end up providing services for free and, in addition, patients complain since their problems are not resolved. These issues involve the availability and the correct use of resources in the health budget.

Consequently, the term "pharmacoeconomics" has been applied more and more frequently. More than simply assigning a monetary value to a given medical intervention, pharmacoeconomics is a relatively recent "knowledge field" that aggregates economic analyses to the common targets of health intervention (for instance safety and efficacy).

Conceptually, pharmacoeconomics identifies and attributes monetary dimensions to health interventions comparing their results to the results of similar interventions and the results of different interventions (which could well be the simple observation of health status). So, pharmacoeconomics allows the determination of the health gain that can be achieved after investing a specific amount of resources. In other words, pharmacoeconomics provides scientific criteria for decisions on the necessary amount of resources for a given area, during a given time, to provide more efficient and rational money allocation [1].

New diagnostic and treatment tools are continuously being developed. It is noteworthy that the FDA has approved 27 new chemical/biological entities for marketing during the 90 's,

Received on 22 October 2005; revised 03 February 2006.

Address for correspondence:Dr.Fernando Franciosi Tatsch. Gerente médico de Divisão de Virologia/TX Roche. Phone: (5511) 37199351.E-mail: fernando.tatsch@ roche.com

The Brazilian Journal of Infectious Diseases 2006;10(1):51-54. (C) 2006 by The Brazilian Journal of Infectious Diseases and Contexto Publishing. All rights reserved. on a yearly basis. The incorporation of pharmaceutical technology during the last years of the $20^{\text {th }}$ century has increased the life expectancy of the American population by 4.7 months [2]. Nevertheless, the development of this technology is highly complex. Only $20 \%$ of the molecules that reach the phase of human testing effectively obtain a license for marketing. In addition, the estimated investment required to obtain a "winner" molecule, among the thousands that are thrown away at early steps, is about 800 million dollars [2]. Therefore, technological development is at the same time necessary and expensive. In conclusion, pharmacoeconomic analysis is particularly useful to managers who need an overall view of new technologies within the list of available health care procedures.

\section{Hepatitis C}

Information regarding the prevalence of hepatitis $C$ virus infection (HCV) in Brazil generally comes from studies that were conducted on potential blood donors. This population surely does not adequately represent the general population, since nearly all the individuals pertaining to the HCV risk group are previously excluded by questionnaires. Indeed, these studies show HCV prevalence in individuals who do not belong to risk groups. There has been, however, a population-based study developed in the city of São Paulo [3]. In this evaluation, samples were collected randomly during domiciliary visits. A prevalence of $1.42 \%$ of positive anti-HCV tests (ELISA) was identified. There is no methodological justification for the extrapolation of this prevalence to the overall Brazilian population. However, If we hypothetically consider this prevalence the same prevalence seen in the Brazilian urban population, which according to IBGE (Brazilian Statistics Institute) is 140 million people [4], we would have about two million people with a positive antiHCV result.

More accurate information can be found in developed countries, such as the United States, where this disease has been studied for many years. In 2000, according to 'United 
States Surgeon General' recommendations, hepatitis C was considered a "silent epidemic" [5]. It is estimated that 3.9 million Americans have been infected by $\mathrm{HCV}$, and among them, 2.7 million (74\%) have developed chronic infection. Currently more than a third of all liver transplantation candidates have hepatitis C infection as the etiology for their hepatic disease. In 1998, approximately $2 \%$ of all hospital admissions (140 thousand people) were related to hepatitis $\mathrm{C}$ complications. This fact lead to health system resources usage that reached about one billion dollars [5-8].

It became evident that there is need for building an "equation" that includes variables such as the resources used to treat cirrhosis and its complications, the impact on patient survival and quality of life and the investments to treat chronic hepatitis. Pharmacoeconomics is the field in which this complex "equation" is built.

\section{Rationale and Tools Used in Pharmacoeconomics}

Overall, it is possible to summarize the targets of health interventions as two main "endpoints": increased longevity and quality of life improvement. The greatest challenge for pharmacoeconomics then becomes clear: how to assess interventions in which gain or loss in the direction of these two endpoints occurs in a non-proportional way. For example, the use of resources by an intensive care unit can enhance the survival of patients with serious stroke with permanent sequels and can also enhance the survival of patients who are treated for leukemia. The quality of life associated with these two different interventions is clearly distinct. Probably, patients who were cured after leukemia treatment will have a quality of life very similar to that observed in the general population. On the other hand, patients with neurological sequels will suffer a significant decrease in their quality of life. So, within a finite time frame, it is possible that these two interventions consume approximately the same amount of resources and generate similar endpoints in survival enhancement, but the quality of life endpoints are quite different.

There are, within the area of pharmacoeconomics, tools and models whose purpose is to unify these two main endpoints in health. In a simplistic way, it is reasonable to say that these models pretend a "standard measurement unit". In other words, through a sophisticated methodology, gains in health are compared, but only after the populations that are studied have been homogenized to a "reference status" of quality of life. The most frequently used unit is "qualityadjusted life years' (QALY) [9]. Each QALY unit gained after a given intervention compared to other intervention means an enhancement in survival without any loss in the quality of life (or with equivalent gains, or equivalent losses, in the quality of life).

Ideally, the pharmacoeconomic models compare the total value of resources that were invested in medical interventions. Thus, when a pharmacoeconomic analysis examines the various alternatives for the treatment and/or follow-up of chronic hepatitis $\mathrm{C}$, medication cost is one variable, but surely, not the only one, or the most important one. Necessarily, all associated costs should be included in the model, such as expenses with transportation, medical visits, complementary exams, work absenteeism, etc. Also, in the case of a simple follow up, the expenses associated with routine evaluations and with disease progress should also be included in the model. Brazil does not have a homogenous pricing reference for such complementary expenses. For example, values attributed to the treatment (or follow up) of chronic hepatitis $\mathrm{C}$ patients differ when one examines a reference center routine in a major city versus a countryside practice.

The 'American Association for the Study of Liver Diseases' recommends, as a primary choice for the treatment of genotypes 1 and non- 1 naïve patients, a combination of peginterferon alfa and ribavirin [10]. In a registration study, peginterferon alfa-2b (12KD) $(1.5 \mathrm{mcg} /$ week $)$, combined with ribavirin, showed a sustained virological response rate of $54 \%$ (versus $47 \%$ for conventional interferon and ribavirin, $\mathrm{P}=0.01$ ) [11]. Peginterferon alfa-2a (40KD) was assessed, at a fixed dose of $180 \mathrm{mcg} /$ week, in two registration trials. Fried et al. [12] have reported $56 \%$ sustained virological response with the combination of peginterferon alfa- $2 \mathrm{a}(40 \mathrm{KD})$ plus ribavirin (versus $44 \%$ for conventional interferon and ribavirin, $\mathrm{P}<0.001$ ). Hadziyannis et al. [13], using this same combination, found an overall sustained virological response of $63 \%$. Therefore, the superiority of peginterferon combined with ribavirin for the treatment of chronic hepatitis $\mathrm{C}$ has been clinically established.

This article reviews the increments in QALYs and eventual increments in the costs associated with the treatment of chronic hepatitis $\mathrm{C}$ with the combination of peginterferon alfa$2 \mathrm{a}(40 \mathrm{KD})$ and ribavirin versus the combination of conventional interferon and ribavirin. The evaluation was conducted outside Brazil. It is a cost-effectiveness analysis in which the cost of the intervention (treatment of hepatitis C) in monetary units was divided by a non-monetary unit (improved survival in QALYs). It is necessary, however, to emphasize that the extrapolation of pharmacoeconomic data is methodologically incorrect, as the values of medical interventions and expenses associated with a given health status vary among different regions and different countries. Finally, it is important to keep in mind that a new medical intervention that enhances survival could also be more expensive. Therefore, it is often necessary to invest even more resources to gain QALYs. Hence it is very important to have societies discussing and clearly defining how much they are willing to "pay" for the additional QALYs. Otherwise, the simple observation that a given treatment results in one additional QALY, compared to some previous treatment at a known cost, does not allow any conclusion, since interventions involving other diseases were not assessed and society has not defined an acceptable value for each additional QALY. 


\section{Pharmacoeconomic Analysis Applied to the Treatment of Chronic Hepatitis C}

Sullivan et al. [14]. examined the cost-effectiveness relation of the combination of peginterferon alfa-2a (40KD) and ribavirin in the United States. This analysis was developed under the perspective of the health provider (managed care association or government, for example) and the intervention selected for comparison was conventional interferon combined to ribavirin. Sustained virological response (SVR) and progression rates of chronic hepatitis $\mathrm{C}$ were obtained from published studies (SVRs already presented in this article). Quality of life information was obtained from medical publications that have used the Health Utility Index tool. Patients who achieved SVR were considered as lifelong HCV free.

The authors have created a flowchart for chronic hepatitis progression within its natural history (Figure 1). This model, conceptually named a 'Markov Model', assumes that the patients remain for finite times in a given status (Markov status) moving on to the following status according to a known yearly probability. For every status, there is a given value of quality of life, which worsens along with the evolution to a more advanced Markov status (Table 1). It was previously admitted that the patients presenting SVR have an interruption in their evolution to the next Markov status, which has a positive impact on survival. Also, whenever a given patient remains within a Markov status with better rates of quality of life, this patient is contributing to an average improvement in the quality of life of the population.

The effectiveness parameter used to evaluate survival improvement was QALY. For a given patient, the QALY was the result of the multiplication of his/her quality of life score (observed in a specific Markov status) by the length of time during which this given patient remained in this specific status. The final result was the sum of all QALY values found, respectively, in each Markov status that the patient has gone through. For example, if a patient remained for 10 years in the status "chronic hepatitis C", whose quality of life score is 0.82 and then remained for five years in the status "compensated cirrhosis", whose quality of life score is 0.78 , then, at the end of these time frames, this patient would have 12.1 QALYs (as a result of [10 X 0.82$]+\left[\begin{array}{l}5 \\ X\end{array} 0.78\right]$ ). Hypothetically, if this same patient had presented a SVR during the status "chronic hepatitis C", he/she would not have progressed to the status "compensated cirrhosis", remaining during all the 15 years time frame without any deterioration in his/her quality of life (possibly, even presenting improvement). Consequently, this patient would have 12.3 QALYs, instead of 12.1 QALYs (as a result of the multiplication of $15 \mathrm{X} 0.82$ ).

As a primary end point, the authors examined the impact on survival observed with peginterferon alfa-2a (40KD) combined to RBV, versus conventional interferon, also
Figure 1. Markov model for chronic hepatitis $\mathrm{C}$ progression. Adapted from Sullivan et al. [14]

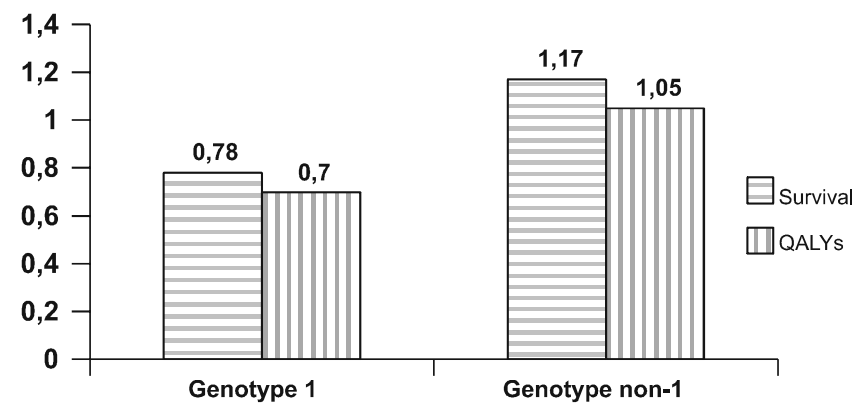

Table 1. Quality adjusted life years scores [14]

\begin{tabular}{ll}
\hline Health-related quality of life score (HUI-Mark III) \\
\hline Chronic hepatitis C & 0.82 \\
Compensated cirrhosis & 0.78 \\
Uncompensated cirrhosis & 0.65 \\
Hepatocarcinoma & 0.25 \\
Liver transplantation & 0.5 (first year) \\
& 0.7 (after the first year) \\
\hline
\end{tabular}

combined with RBV, in the treatment of chronic hepatitis $\mathrm{C}$ patients. The estimated average survival for patients treated with conventional interferon/RBV was 29.23 years. Genotype 1 patients treated with peginterferon alfa- $2 \mathrm{a}(40 \mathrm{KD}) / \mathrm{RBV}$ had an increase of 0.78 years in the average survival rate, when compared to patients treated with conventional interferon/ RBV. The increase in survival of genotype non-1 patients was 1.17 years. These gains in survival meant an increase of 0.7 and 1.05 QALYs in genotype 1 and non-1, respectively (Figure $2)$. The additional cost to treat patients with peginterferon alfa- $2 \mathrm{a}(40 \mathrm{KD}) / \mathrm{RBV}$ is the total cost of peginterferon alfa- $2 \mathrm{a}$ (40KD)/RBV treatment minus the total cost of conventional interferon/RBV treatment.

Finally, the authors divided the additional cost of treating genotype 1 patients with peginterferon alfa-2a (40KD)/RBV by the number of QALYs that were gained. They found that an increase of 1 QALY costs 2,600 dollars, when this population was submitted to the newer therapeutic regimen. In genotype non-1 patients, the regimen of peginterferon alfa$2 \mathrm{a}(40 \mathrm{KD}) / \mathrm{RBV}$ was more effective and decreased the costs (Table 2). Therefore, there was an enhancement of effectiveness, expressed as QALYs at a lower cost, when compared to the conventional interferon/RBV regimen.

\section{Discussion}

An intervention has a favorable cost-effectiveness relation when it does not go beyond the limit established by a given society. In the United States, interventions in which the cost 
of a single QALY is below 16,500 dollars are considered acceptable [1-14]. In the discussed study [14], only genotype1 patients showed a costs increase when treated with peginterferon alfa-2a (40KD)/RBV. However, the cost of 2,600 dollars per QALY is well within the range accepted in that country. In genotype non-1 patients, the survival enhancement expressed as quality-adjusted life years (QALY) was not followed by a costs increase. Therefore, conceptually, this situation is defined as "dominant". In other words, the intervention should be promptly incorporated to the list of available health care procedures.

It is interesting to observe that, when the authors changed arbitrarily, but within a plausible range, some parameters such as the rates of progression among the Markov status or the

Figure 2. Survival and increase in quality of life adjusted years [14]

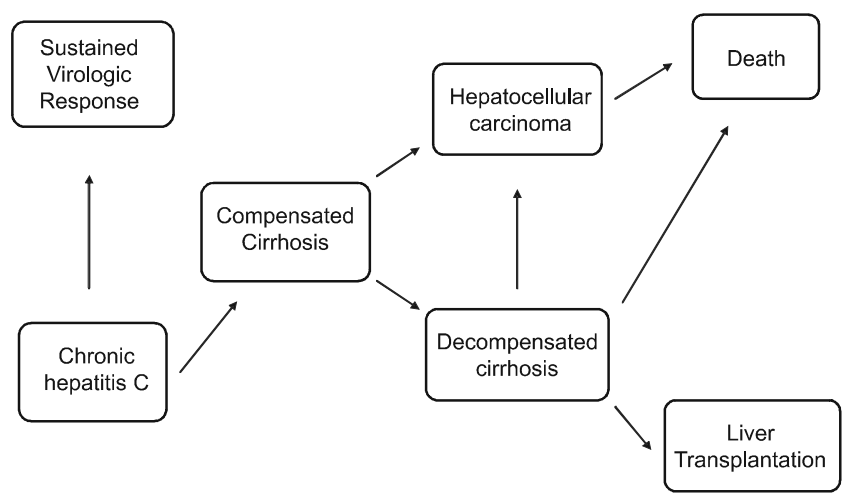

Table 2. Cost per quality adjusted life year (QALY) [14]

\begin{tabular}{ll}
\hline & $\begin{array}{l}\text { Cost per QALY (peginterferon a-2a } \\
(\mathbf{4 0 K D ) / R B V ~ v e r s u s ~} \text { interferon/RBV) }\end{array}$ \\
\hline Genotype 1 & US\$2,600 \\
Genotype non-1 & Dominant* \\
\hline $\begin{array}{l}\text { Cost per QALY with peginterferon a-2a }(40 \mathrm{KD}) / \mathrm{RBV} \text { was lower } \\
\text { than the cost per QALY with conventional interferon/RBV }\end{array}$
\end{tabular}

rates of sustained virological responses, the cost per QALY remained below 16,500 dollars. This analysis tests the sensibility of the results in case of variable changes.

Products and services have different prices in different regions or countries. For this reason pharmacoeconomic analysis must be adapted to a local reality. It is correct to conclude, based on the study analyzed in this review, that the treatment of chronic hepatitis $C$ is cost-effective in the country where the study was conducted. In Brazil, the largest challenge appears to be correct information on the eventual SVR seen with the use of conventional interferon (which is different from the one used by Manns [11] and Fried [12]). Consequently, any comparison of efficacy would be faulty, making it very difficult to make a pharmacoeconomic analysis.

\section{References}

1. Kolbet G. Health Economics - General Issues. In: Kolbet G. Health Economics: An Introduction to Economic Evaluation. 2.ed. London: Office of Health Economics 2002;1:9-24.

2. Scherer F.M. The Pharmaceutical Industry - Prices and Progress. New England Journal of Medicine 2004;351:92732.

3. Focaccia R., Conceição O.J.G, Sette H., et al. Estimated Prevalence of Viral Hepatitis in the General Population of the Municipality of São Paulo Measured by a Serologic Survey of a Stratified, Randomized and Residence-Based Population. Brazilian Journal of Infectious Desease 1998;2(6):269-84.

4. IBGE. Censo Demográfico 2000 - Resultados do universo. Available at http://www.ibge.gov.br, accessed on 28/nov/ 2004.

5. Kim W.R. The Burden of Hepatitis $\mathrm{C}$ in United States. Hepatology 2002;36:S30-S4.

6. Lauer G.M., Walker B.D. Hepatitis C Virus Infection. New England Journal of Medicine 2001;345:41-52.

7. Herrine S.K. Approach to the Patient with Chronic Hepatitis C Virus Infection. Annals of Internal Medicine 2002; 136:74757.

8. Zeu Zem S. Heterogeneous Virologic Response Rates to Interferon-Based Therapy in Patients with Chronic Hepatitis C: Who Responds Less Well? Annals of Internal Medicine 2004; 140:370-81.

9. Meltzer M.I. Introduction to Health Economics for Physicians. Lancet 2001; 358:993-98.

10. Strader D.B., Wright T., Thomas D.L., Seeff L.B. Diagnosis, Management and Treatment of Hepatitis C. Hepatology 2004;39:1147-71.

11. Manns M., Mchutchison J., Gordon S., et al. Peginterferon alfa-2b plus Ribavirin Compared with Interferon Alfa-2b plus Ribavirin for Initial Treatment of Chronic Hepatitis C: a Randomized Trial. Lancet 2001;358:958-65.

12. Fried M., Shiffman M.L., Reddy K.R., et al. Peginterferon Alfa2a (40KD) plus Ribavirin for Chronic Hepatitis C Virus Infection. New England Journal of Medicine 2002;347:97582.

13. Hadziyannis S., Sette H., Morgan T., et al. Peginterferon Alfa-2a (40KD) and Ribavirin Combination Therapy in Chronic Hepatitis C A Randomized Study of Treatment Duration and Ribavirin Dose. Annals of Internal Medicine 2004; 140:346-55.

14. Sullivan S.D., Jensen D.M., Bernstein D.E., et al. Cost Effectiveness of Combination Peginterferon Alfa-2a (40KD) and Ribavirin Compared With Interferon Alfa-2b and Ribavirin in Patients With Chronic Hepatitis C. American Journal of Gastroenterology 2004;99:1490-6. 